\title{
The global prevalence $p t x P 3$ lineage of Bordetella pertussis was rare in young children with the co-purified aPV vaccination: a 5 years retrospective study
}

Zengguo Wang ${ }^{1 *+} \mathbb{D}$, Yang Luan ${ }^{2 \dagger}$, Quanli Du², Chang Shu ${ }^{1}$, Xiaokang Peng ${ }^{1}$, Huijing Wei ${ }^{1}$, Tiejun Hou², Ying Liu², Xiaoguai Liu ${ }^{1^{*}}$ and Yarong $\mathrm{Li}^{\mathrm{i}^{*}}$

\begin{abstract}
Background: The global prevalent ptxP3 strains varies from about $10 \%$ to about $50 \%$ of circulating B. pertussis population in different areas of China.

Methods: To investigate the difference of vaccination status between different genotypes in the circulating $B$. pertussis after 10 years of acellular pertussis vaccine (aPV) used in China. The nasopharyngeal swabs and isolates of B. pertussis from these patients were used to perform genotyping of antigen genes. We use antibiotic susceptibility test against erythromycin and sequencing methods for site 2047 of $23 \mathrm{~S} \mathrm{rRNA}$ to determine the resistance status.

Results: The ptxP1 allele with erythromycin resistant (ER) B. pertussis infection (total of 449 subjects) consisted of 84.70 to $96.70 \%$ from 2012 to 2016 in this study. Vaccinated with co-purified aPV was found in 133(133/403,33.0\%), $1(1 / 9,11.1 \%)$ and $2(2 / 21,9.5 \%)$ in ptXP1/fhaB3-ER, ptXP1/fhaB2-ES and ptXP3/fhaB2-ES B. pertussis infected children each, which showed a significant difference $\left(X^{2}=6.87, P=0.032\right)$.

Conclusions: The ptxP3-ES B. pertussis was rare while the ptXP1-ER B. pertussis was steadily increased in Xi'an, China from 2012 to 2016, where co-purified aPV was prevalent used. This pose a hypothesis that the co-purified aPV might protect against $p t \times P 3$ strains more efficient, which generated a rare chance for ptxP3 strains to be under the antibiotic pressure and further developed to be erythromycin resistance. A further cohort study and the mechanisms of the additional antigen proteins of co-purified aPV protected against B. pertussis should be consideration.
\end{abstract}

Keywords: Bordetella pertussis, Pertussis, Acellular pertussis vaccine, Resistance, Membrane protein

\footnotetext{
*Correspondence: william_wzg@126.com; xiaoguai760725@126.com;

lyr640101@163.com

'Zengguo Wang and Yang Luan contributed equally to this work.

${ }^{1}$ Xi'an Children's Hospital, 69 Xijunyuan Road, Xi'an 710002, Shaanxi Province,

China

Full list of author information is available at the end of the article
}

(c) The Author(s). 2020 Open Access This article is licensed under a Creative Commons Attribution 4.0 International License, which permits use, sharing, adaptation, distribution and reproduction in any medium or format, as long as you give appropriate credit to the original author(s) and the source, provide a link to the Creative Commons licence, and indicate if changes were made. The images or other third party material in this article are included in the article's Creative Commons licence, unless indicated otherwise in a credit line to the material. If material is not included in the article's Creative Commons licence and your intended use is not permitted by statutory regulation or exceeds the permitted use, you will need to obtain permission directly from the copyright holder. To view a copy of this licence, visit http://creativecommons.org/licenses/by/4.0/ The Creative Commons Public Domain Dedication waiver (http://creativecommons.org/publicdomain/zero/1.0/) applies to the data made available in this article, unless otherwise stated in a credit line to the data. 


\section{Background}

Pertussis is a respiratory disease mainly caused by Bordetella pertussis. The incidence of pertussis marked decreased after the whole cell pertussis vaccine (wPV) has introduced all over the world. Since the 1990s, a resurgence of pertussis has appeared in many countries, especially when the acellular pertussis vaccine (aPV) has replaced from the wPV. Furthermore, the circulating B. Pertussis has evolved mainly changed in the vaccine antigen genes proposed by the vaccine-driven, such as the $p t x P 1$ lineage to $p t x P 3$ lineage and also pertactin deficient [1]. Nowadays, the $p t x P 3$ lineage with/or without pertactin deficient strains, which has been proved to be more virulent and reflect selective advantage under the high coverage of aPV vaccination, has emerged globally and raised an important public issue toward an alternative vaccine in pertussis prevention [2].

However, except what happened in some countries like Iran, the $p t x P 1$ lineage was still prevalence in most countries used wPVs [3]. We have reported the $p t x P 1$ strains further shown erythromycin resistance (ER) that emerged in China since 2012. Furthermore, we found that all the ptxP1- ER strains originated from a fhaB3 lineage, which appears to have been selected from the wPV or antibiotic pressure [4]. Interestingly, although the ptxP1-ER strains expanded all the countries of China, the proportions of ptxP3-ES strains varied from less than $10 \%$ to about $50 \%$ in different areas of China, especially occurred much higher in developed areas $[5,6]$.

The aPV came in two varieties according to the producing procedures: one is obtained through co-purified procedures so called co-purified aPV, which was used primarily in China and Japan. The other one with purification of each one to five components individually antigen and then blending them in an appropriate ration called purified aPV, which was used in lots of areas all over the world [7]. In China, the co-purified aPV was free and used predominantly since 2006. The purified aPV (Sanofi) was imported and rechargeable since 2011 and supplied much more in developed areas in China. Despite the ptxP3-ES strains were proved to be adapted to the purified aPV all over the world and also been prevalence under wPV vaccination in Iran $[3,8]$. Moreover, whether the different proportions of ptxP3-ES strains in China were associated with the types of aPV remains enigmatic.

In this study, we conducted a 5-year retrospective study to survey the dynamic changes in genetic makeup \& resistance status of the circulating $B$. pertussis and further the difference in demographic characteristics between different genotypes in Xi'an China, where copurified aPV was still prevalence used. We are kind of hoping studies such as this can give more information in consideration of the modified vaccine for global pertussis prevention.

\section{Methods}

\section{Study populations, strains and samples}

All the patients admitted to Xi'an Children's Hospital for suspected of pertussis from 2012 to 2016 were sampled of nasopharyngeal swabs (NPs) and diagnostic by culture and special PCR for B. pertussis as previous reported [9, 10]. The DNAs from B. pertussis ATCC 9797 and $\mathrm{ddH}_{2} \mathrm{O}$ were used as positive and negative control in each PCR run [9]. The demographic characteristics were collected if culture and/or special PCR for B. pertusssis was positive. Totally, 702 cases were positive by the special PCR which including 204 cases with both culture and PCR positive of $B$. pertussis. All the strains and NPs were stored at $-80^{\circ} \mathrm{C}$ until to use.

\section{Antibiotic susceptibility test}

In-vitro sensitivity of clinical strains against erythromycin was performed and reported as previously [9]. We used B. pertussis ATCC 9797 and Staphylococcus aureus ATCC 25923 as controls.

\section{$23 \mathrm{~S}$ rRNA sequencing and antigen gene typing}

The nucleotide position 2047 of the 23S rRNA was performed by DNAs of strains and/or NPs by our previously reported sequencing methods [9]. Cause the A2047G of 23S rRNA was associated with erythromycin resistance, if the nucleotide position 2047 of the 23S rRNA was the wild type as adenine (A), we defined as an erythromycin sensitive $B$. pertussis infection. A mutation type as guanine (G) of site 2047 was taken for erythromycin resistance $B$. pertussis infection strain [11, 12]. The allele of $p t x P$ and $f h a B$ was performed by DNAs of strains and/or NPs as previously reported when successful sequencing of $23 \mathrm{~S}$ rRNA [13].

\section{Statistical analysis}

Data were statistically analyzed with SPSS 17.0. Comparisons were performed using $X^{2}$ test or one-way analysis of variance (ANOVA). A $P$ value $<0.05$ was considered statistical significant.

\section{Results}

In total of 204 isolates, 3 strains were ptxP3 allele and the others were all $p t x P 1$ alleles. Four strains, including of $3 p t x P 3 / f h a B 2$ and $1 p t x P 1 / f h a B 2$ allele, have the MICs against erythromycin lower than $0.023 \mu \mathrm{g} / \mathrm{ml}$, which refers to sensitive to erythromycin in vitro. The rest of the 200 strains were all resistant to erythromycin with the MICs $\geq 256 \mu \mathrm{g} / \mathrm{ml}$, which were all $p t x P 1 / f h a B 3$ allele. All the resistant strains posed an A2047G mutation in 23S rRNA and no mutation occurred this site of the sensitive strains.

Among the 702 NPs for sequencing, 480 obtained both the available sequencing results of 23 rRNA, $p t x P$ and 


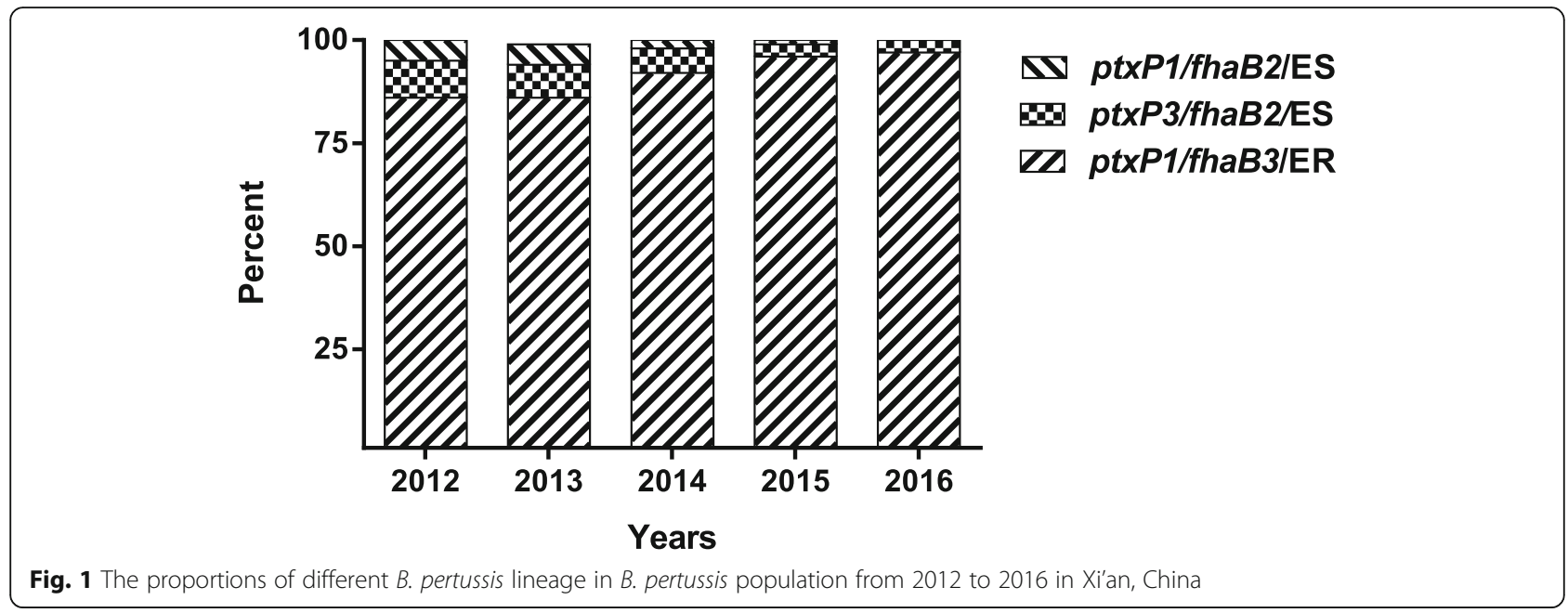

fhaB. The NPs generated the sequencing results as same as obtained by strains from the same patient. There were 449 in 480 specimens (93.5\%) shown the allele G in 2047 site of $23 \mathrm{rRNA}$ that defined as erythromycin resistant $B$. pertussis infection, which also shown the allele of ptxP1/ fhaB3. The dynamic changes of proportions of circulating B. pertussis from 2012 to 2016 as shown in Fig. 1.

Furthermore, 47 patients were excluded when analysis the difference among demographic characteristics cause of unclear vaccination status. Among the remaining 433 patients, 136 patients have been administrated with least 1 does of co-purifid aPV. Vaccinated with co-purified aPV was found in $133(133 / 403,33.0 \%), 1(1 / 9,11.1 \%)$ and $2(2 / 21,9.5 \%)$ in $p t x P 1 / f h a B 3-E R, p t x P 1 / f h a B 2-E S$ and ptxP3/fhaB2-ES infected children, which showed a significant difference $\left(X^{2}=6.87, P=0.032\right)$. (Table 1 ).

\section{Discussion}

Within our study, we discovered that ptxP1-ER strains have been steadily increased to the circulating $B$. pertussis population from 2012 to 2016 in Xi'an, China. Moreover, unlike that $B$. pertussis could not only infect the infants that were too young to be vaccinated, but also the infants vaccinated with the purified aPV $[14,15]$, the ptxP3 strains rarely infected the infants administrated with co-purified aPV from our study.

The ptxP3 strains have been circled predominant all over the world, especially after the replacement of wPV by aPV. However, the ptxP3 strains have spread across the globe seems not only driven by aPV selection, but also by the fitness of ptxP3 strains when compete with erythromycin sensitive non- ptxP3 strains $[3,8]$. Furthermore, we assumed that the quality of the wPV between different batch and/or the frequency of international movement of people possibly accelerated the prevalence of ptxP3 strains in Iran too. The increasing incidence of pertussis was also emerged in China from 2013 according to the national infectious diseases case reported system. Besides the A2047G mutation in 23S rRNA occurred in ptxP1-ER B. pertussis strains, a novel fhaB C5330T was also founded in all these strains but didn't appear in any ptxP3 lineage. This fhaB3 lineage has been proved to be prevalence among China via expansions most likely due to vaccine and/or antibiotic pressure [4]. This study illustrated that the ptxP1/fhaB3-ER

Table 1 The demographic characteristics of children suffering from pertussis with different genetic makeup \& erythromycin resistance status of $B$. pertussis

\begin{tabular}{|c|c|c|c|c|c|}
\hline & ptxP1/fhaB3-ER & ptxP3/fhaB2-ES ${ }^{a}$ & ptxP1/fhaB2-ES & $x^{2}$ & $P$ \\
\hline & $n=403(\%)$ & $n=21(\%)$ & $n=9(\%)$ & & \\
\hline Age (Months) ${ }^{b}$ & $3(2-5.5)$ & $2(1-3.5)$ & $3(2-5)$ & $1.479^{c}$ & 0.225 \\
\hline Vaccination status $^{d}$ & & & & 6.87 & 0.032 \\
\hline Vaccinated & $133(33.0)$ & $2(9.5)$ & $1(11.1)$ & & \\
\hline Unvaccinated & $270(67.0)$ & $19(90.5)$ & $8(88.9)$ & & \\
\hline
\end{tabular}

${ }^{a}$ The ptxP3-ES with proportions of $8.93,9.38,6.19,2.65$ and $3.09 \%$ from 2012 to 2016 in this study

${ }^{\mathrm{b}}$ The ages were represented as Med, $\mathrm{x} .5$ (Q1, X.25- Q3, X.75)

${ }^{c}$ Refers to the $\mathrm{F}$ value with ANOVA test between ptxP1-ER and ptxP3-ES group

${ }^{\mathrm{d}}$ The cases of unclear vaccination status were not enrolled, what was 46 and 1 in ptxP1-ER and ptxP3-ES group each. All the vaccinated group administrated with the Co-aPV 
strains might be adapted to the co-purified aPV more easily than global $p t x P 3$ strains.

It was reported that the $p t x P 3$ strains have been occurred in 2000 and remains sporadic in this country [16]. According to this study, $p t x P 3$ strains with the decreased proportions have observed from 2012 to 2016 in Xi'an, the western of China. In China, the co-purified aPV was free and predominated used since 2006 while the purified aPV (Sanofi) was available by paid since 2011with rarely market supplied, especially in undeveloped regions of western China, such as Xi'an. The rare of $p$ txP3 strains in Xi'an after 10 years of co-purified aPV used might give a clue that the co-purified aPV did not give the adaption as purified aPV did in developed countries where $p t x P 3$ was quickly predominant worldwide [8]. The co-purified aPVs have more protein antigen than purified aPVs [17]. Therefore, this study further supported the hypothesis that the small antigen targets of purified aPV could induce the vaccine pressure and vaccine adaption more easily than the more antigen targets vaccine, such as $\mathrm{wPV}$, even the co-purified aPV [18]. Furthermore, among the additional protein antigens of co-purified aPV, most was the out membrane proteins such as BipA and SphB1. Such membrane proteins containing in the outer membrane vesicles (OMVs) of $B$. pertussis have been suggested as an attracting candidate component of the possible new modified vaccine against pertussis $[19,20]$. The latest study further proved that the OMVs can protect against B. pertussis with long term duration, even the global popular $p t x P 3$ and pertactin deficient strains [20]. Japan was the first country to develop aPV (co-purified) in 1981 and to adopt for use in the general population. It has been reported that both of the two types of aPVs was used recently [21]. However, the ptxP3 lineage still holds lower than 50\% from 2006 to 2010 until the period of 2011-2014which reached close to $80 \%$ [22].

Most of the cases in this study were from the west of China. Otherwise, it was reported that the ptxP1-ER strains contributed to $75.4,50.7$ and $48.6 \%$ in the circulating strains in Zhejiang province (Southern of China, 2016), Shanghai (Southern of China, 2016-2017) and Shenzhen (Southern of China,2015-2017), while the rest ES strains were almost $p \operatorname{txP} 3$ strains $[5,23,24]$. No details of the vaccine type were described in these relative high proportion of ptxP3 areas of China. Liking what happened to Japan, we assumed that the purified aPV used was much more in these developed areas of China than in Xi'an, which generate a relative low level of vaccine protection from co-purified aPV in general population. As a result, the proportions of $p \operatorname{txP} 3$ strains were much more.

Consistent with reports in these areas of China, the erythromycin resistant strains were all $p t x P 1$ allele while the $p t x P 3$ strains were all sensitive to erythromycin. As shown in this study, though the average age of ptxP3-ES strains infection group is lower than in $p t x P 1-E R$ groups, no statistic significant difference was observed. Furthermore, more than $85 \%$ of subjects have taken antibiotics before sampling and detection, no difference was observed between the ptxP3- ES and ptxP1-ER groups (data not shown). Therefore, despite the antibiotic pressure which seems to provide the selective advantage for expansion of erythromycin resistant strains, we pose a hypothesis that the co-purified aPV protect against ptxP3 strains more efficient, which generated a rare chance for $p \operatorname{txP} 3$ strains to be under the antibiotic pressure and further developed to be erythromycin resistance.

However, It is a limitation that the cases of $p t x P 3$ strains were relative too small to give strong evidence about the protection against $p t x P 3$ lineage by co-purified aPV. Furthermore, the ptxP3 with the pertactin (PRN) deficient isolates were widely appeared in some industries countries [25], whether the ptxP3 isolations in this study expressed of PRN were unknown in this study. Lastly, the age of the patients in our study was mainly the infant but not children after at least 5 years of vaccination of co-purified aPV. Thus we can not give powerful support about the protection duration against ptxP3 linage of the co-purified aPV.

\section{Conclusions}

In conclusion, this study revealed that the erythromycin resistant $B$. pertussis have been steadily increased from 2012 to 2016 in Xi'an, western of China. We also pose a hypothesis that the co-purified aPV containing more antigens has the possibility to protect the infant from being ill of pertussis infected by global popular $p t x P 3$ lineage B. pertussis. To be better understanding the effect of copurified aPV, an international multicenter cohort study should be performed.

\section{Abbreviations \\ aPV: Acellular pertussis vaccine; ER: Erythromycin resistant; wPV: Whole cell pertussis vaccine; NPs: Nasopharyngeal swabs; OMVs: Outer membrane vesicles; PRN: Pertactin}

\section{Acknowledgements}

Not applicable.

\section{Authors' contributions}

ZW, $\mathrm{XL}$ and $\mathrm{YL} 3$ contributed to the study design. YL1 and QD contributed to carry out the experimental work, writing and data analysis. CS, XP and HW contributed to acquisition of data. YL2 and TH collected the data and samples. All authors reviewed and approved the final approved the final manuscript as submitted.

\section{Funding}

This study is supported by National Nature Science Foundation of China (No.81602902) mainly with the design of the study, the project of Xi'an postdoctoral innovation foundation (2019-10) with collection, analysis and 
interpretation of data, and the project of Xi'an Children's Hospital (2019A02) with the manuscript writing.

\section{Availability of data and materials}

Xi'an Children's hospital is the custodian of the data for this study. The data are not accessible online, but may be made available upon written request to the authors, if in line with the Ethical Review Board guidelines.

\section{Ethics approval and consent to participate}

This study was approved by the institutional Review Board of Xi'an Children Hospital, Xi'an, Shannxi Province, China. All the data used was anonymised.

\section{Consent for publication}

Not applicable.

\section{Competing interests}

All the authors declare that they have no competing interests.

\section{Author details}

${ }^{1}$ Xi'an Children's Hospital, 69 Xijunyuan Road, Xi'an 710002, Shaanxi Province, China. ${ }^{2}$ Xi'an Center for Disease Control and Prevention, 599 Xiying Road, Xi'an 710054, China.

Received: 10 February 2020 Accepted: 10 August 2020

Published online: 19 August 2020

\section{References}

1. Melvin JA, Scheller EV, Miller JF, Cotter PA. Bordetella pertussis pathogenesis: current and future challenges. Nat Rev Microbiol. 2014;12(4): 274-88

2. Allen A. Public health. The pertussis paradox. Science. 2013;341(6145):454-5.

3. Safarchi A, Octavia S, Nikbin VS, Lotfi MN, Zahraei SM, Tay CY, Lamichhane B, Shahcheraghi F, Lan R. Genomic epidemiology of Iranian Bordetella pertussis: 50 years after the implementation of whole cell vaccine. Emerg Microbes Infect. 2019;8(1):1416-27.

4. Xu Z, Wang Z, Luan Y, Li Y, Liu X, Peng X, Octavia S, Payne M, Lan R. Genomic epidemiology of erythromycin-resistant Bordetella pertussis in China. Emerg Microbes Infect. 2019;8(1):461-70.

5. Zhang JS, Wang HM, Yao KH, Liu Y, Lei YL, Deng JK, Yang YH. Clinical characteristics, molecular epidemiology and antimicrobial susceptibility of pertussis among children in southern China. World J Pediatr. 2019;16(2): 185-92.

6. Yang Y, Yao K, Ma X, Shi W, Yuan L. Variation in Bordetella pertussis susceptibility to erythromycin and virulence-related genotype changes in China (1970-2014). PLoS One. 2015;10(9):e0138941.

7. Wang L, Lei D, Zhang S. Acellular pertussis vaccines in China. Vaccine. 2012; 30(50):7174-8

8. Bart MJ, Harris SR, Advani A, Arakawa Y, Bottero D, Bouchez V, Cassiday PK Chiang CS, Dalby T, Fry NK, et al. Global population structure and evolution of Bordetella pertussis and their relationship with vaccination. mBio. 2014; 5(2):e01074.

9. Wang Z, Cui Z, Li Y, Hou T, Liu X, Xi Y, Liu Y, Li H, He Q. High prevalence of erythromycin-resistant Bordetella pertussis in Xi'an, China. Clin Microbiol Infect. 2014;20(11):0825-30.

10. Tatti KM, Sparks KN, Boney KO, Tondella ML. Novel multitarget real-time PCR assay for rapid detection of Bordetella species in clinical specimens. J Clin Microbiol. 2011:49(12):4059-66.

11. Bartkus JM, Juni BA, Ehresmann K, Miller CA, Sanden GN, Cassiday PK, Saubolle M, Lee B, Long J, Harrison AR Jr, et al. Identification of a mutation associated with erythromycin resistance in Bordetella pertussis: implications for surveillance of antimicrobial resistance. J Clin Microbiol. 2003:41(3):1167-72

12. Wang Z, Han R, Liu Y, Du Q, Liu J, Ma C, Li H, He Q, Yan Y. Direct detection of erythromycin-resistant Bordetella pertussis in clinical specimens by PCR. J Clin Microbiol. 2015;53(11):3418-22

13. Litt DJ, Jauneikaite E, Tchipeva D, Harrison TG, Fry NK. Direct molecular typing of Bordetella pertussis from clinical specimens submitted for diagnostic quantitative (real-time) PCR. J Med Microbiol. 2012;61(Pt 12): $1662-8$
14. Goldstein E, Worby CJ, Lipsitch M. On the Role of Different Age Groups and Pertussis Vaccines During the 2012 Outbreak in Wisconsin. Open Forum Infect Dis. 2018;5(5):ofy082

15. Skoff TH, Hadler S, Hariri S. The Epidemiology of Nationally Reported Pertussis in the United States, 2000-2016. Clin Infect Dis. 2018;68(10):163440.

16. Xu Y, Liu B, Grondahl-Yli-Hannuksila K, Tan Y, Feng L, Kallonen T, Wang L, Peng $D$, He $Q$, Wang $L$, et al. Whole-genome sequencing reveals the effect of vaccination on the evolution of Bordetella pertussis. Sci Rep. 2015;5: 12888.

17. Xu Y, Tan Y, Asokanathan C, Zhang S, Xing D, Wang J. Characterization of co-purified acellular pertussis vaccines. Hum Vaccin Immunother. 2015;11(2): 421-7.

18. Kennedy DA, Read AF. Why does drug resistance readily evolve but vaccine resistance does not? Proc Biol Sci. 2017;284(1851):20162562.

19. Hozbor DF. Outer membrane vesicles: an attractive candidate for pertussis vaccines. Expert Rev Vaccines. 2017;16(3):193-6.

20. Zurita ME, Wilk MM, Carriquiriborde F, Bartel E, Moreno G, Misiak A, Mills KHG, Hozbor D. A pertussis outer membrane vesicle-based vaccine induces lung-resident memory CD4 T cells and protection against Bordetella pertussis, Including Pertactin Deficient Strains. Front Cellular Infect Microbiol. 2019;9:125.

21. Okada K, Komiya T, Yamamoto A, Takahashi M, Kamachi K, Nakano T, Nagai T, Okabe N, Kamiya H, Nakayama T. Safe and effective booster immunization using DTaP in teenagers. Vaccine. 2010;28(48):7626-33.

22. Zomer A, Otsuka N, Hiramatsu Y, Kamachi K, Nishimura N, Ozaki T, Poolman J, Geurtsen J. Bordetella pertussis population dynamics and phylogeny in Japan after adoption of acellular pertussis vaccines. Microb Genom. 2018; 4(5):e000180.

23. Hua CZ, Wang HJ, Zhang Z, Tao XF, Li JP, Mi YM, Tang LF, Chen ZM. In vitro activity and clinical efficacy of macrolides, cefoperazone-sulbactam and piperacillin/piperacillin-tazobactam against Bordetella pertussis and the clinical manifestations in pertussis patients due to these isolates: a singleCentre study in Zhejiang Province, China. J Glob Antimicrob Resist. 2019;18: 47-51.

24. Fu P, Wang C, Tian H, Kang Z, Zeng M. Bordetella pertussis infection in infants and young children in Shanghai, China, 2016-2017: clinical features, genotype variations of antigenic genes and macrolides resistance. Pediatr Infect Dis J. 2019:38(4):370-6.

25. Barkoff AM, He Q. Molecular epidemiology of Bordetella pertussis. Adv Exp Med Biol. 2019;1183:19-33.

\section{Publisher's Note}

Springer Nature remains neutral with regard to jurisdictional claims in published maps and institutional affiliations.

Ready to submit your research? Choose BMC and benefit from:

- fast, convenient online submission

- thorough peer review by experienced researchers in your field

- rapid publication on acceptance

- support for research data, including large and complex data types

- gold Open Access which fosters wider collaboration and increased citations

- maximum visibility for your research: over $100 \mathrm{M}$ website views per year

At BMC, research is always in progress.

Learn more biomedcentral.com/submissions 